mercury three weelss. 3. A patient in the Lock Hospital was suffering with a hard sore, a bubo, and a general tubercular syphilide. He had taken no mercury. With his consent a papule with the subjacent cutis was excised with scissors from the front of the thorax, the skin being previously cleansed with a solution of carbolic acid $(1$ in 20 ), and the scissors heated immediately before use. A blood-serum tube was at once inoculated from the lymph exuding from the base of the papule, and in the course of a few days a cultivation similar to the preceding developed. On subsequent examination sections of the papule showed very numerous bacilli scattered in the connective tissue interspaces of the corium and around and within the small aggregations of round cells, which in parts invested the capillaries. Many of the bacilli showed the unequal staining to be presently described; others were uniformly stained (see Fig. 1). 4. A similar organism was also obtained in a cultivation from a much enlarged and distinctly indurated lymphatic gland from a man who had indurated but somewhat ulcerated sore in the furrow

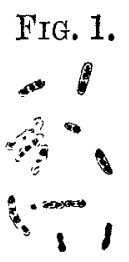

Bacill in sections of a syphilitic papule. The three pale bacilli were unstained, and are possibly bacilli containing and are possibly bacild
spores (950 diameters).

around the glans penis. The sore and the glans were axcised at the same time, and in both bacilli were demonstrated. The sore had existed for one month. He left the hospital shortly after the operation and was subsequently lost sight of. The condition of the glands, the histological characters of the sore, and the history of the case, were all in favour of the view that he had syphilis. 5. Cultivation from lymph exuding from the base of a somewhat inflamed sore on the inner surface of the prepuce, which was removed by circumcision. There were distinctly indurated glands in both groins, and the patient subsequently developed secondary manifestations. 6. An impure cultivation grew after inoculation with lymph from the base of a freshly excised condyloma.

In none of the cases from which cultivations were obtained had mercury been administered for any length of time, and a long series of failures have led us to reject entirely cases which have been long under mercurial treatment. All these cultivations were composed of a very peculiar and somewhat polymorphous micro-organism (see Fig. 2), consisting of longer or shorter rod-like bodies, with rounded, and
FIG. 3.

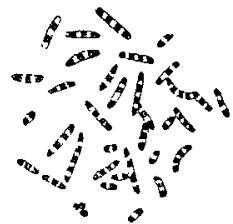

Bacilli in section of a chancre (950 diameters).
FIG. 4.

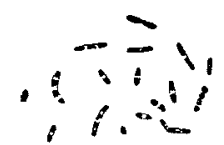

Cultivation of bacilli from a phagedænic sore (950 diameters). sometimes enlarged, or distinctly club-shaped ends; they were usually straight, but occasionally curved. The longer bacilli were as a rule unequally stained, and showed from three to five, or even eight, deeply stained segments united by unstained protoplasm and enclosed in a hyaline sheath. Organisms were observed in which the unstained material was drawn out in a thread-like filament connecting the enlarged end to the rest of the bacillus. The shorter organisms were composed of two elongated or oval masses of stained protoplasm separated by a clear interval, which relatively increased as the bacillus became longer. The transitional forms from these to the long beaded bacilli were patent to the observer. If the specimens after staining were not well washed, the organisms were uniformly coloured, or the beading was indistinct; hence in sections this peculiar characteristic could not be so clearly brought out; but it is well shown in Fig. 3, which was taken from a chancre in an undoubted case of syphilis. Many of the bacilli in sections of this sore were uniformly stained, or showed a clear median space such as may be seen in the short and in the uniformly stained bacilli in Figs. 1 and 2. Other slight shades of difference may be explained by the varying conditions under which the organisms grow in culture media and in the various textures of the body. The bacilli in the case above referred to existed in great abundance, either singly or in groups, in the connective tissue interspaces around and at the base of the sore; they were less numerous in the granulation tissue, a fact whlch may be explained by the observations of Ribbert on the resisting power of leucocytes to the growth of micro-organisms. This was their usual mode of distribution in chancres, but they were in many insiances observed in lymph capillaries and within large clear cells. In lymphatic glands the bacilli were most abundant within and in the tissues adjoining the peripheral lymph channels, and were observed also in lymph cells. In sections of condyloma they were numerous in the connective tissue interspaces of the corium, still more so throughout the epithelium, and formed colonies on the surface. In gummata they were most numerous at the margins, where the disease was extending. In a case of phagedænic sore, a cultivation (see Fig. 4) closely resembling that above described was obtained from the ichor flowing from its surface, and later from the lymph flowing from the base of the sore after its free excision. Induration of the inguinal glands on both sides subsequently appeared, and syphilis was diagnosed. The general features and the staining reaction of this bacillus were the same as in that found in ordinary cases of syphilis, but the segmentation or beading was not so well marked. The cultivations obtained from syphilitic patients appeared as a thin, faintly yellow or brownish yellow, uniform, or slightly tuberculated layer on the surface of the media. The organism grew in solidified blood-serum, which it did not liquefy, and less readily in solidified hydrocele fluid and agar-agar. As regards its differentiation from the bacillus described by Alvarez and Tavel, as existing in normal smegma, we find that, in contradistinction to the latter, the bacillus here described is, after staining with fuchsin, decolourised by dilute nitric acid. Decolourisation occurs both in cultivations and in sections, and is also accomplished by oxalic acid. We have studied the bacillus of normal smegma both in scrapings and by cultivations, and are unable to identify it with that which we have found in syphilis. Nor does it seem in the least degree probable that in syphilis a non-pathogenic organism should be found in all the lesions, even in the internal organs. In sections known to be crowded with bacilli uniformly negative results have followed the use of Lustgarten's method. Our best preparations have been obtained by staining with a solution of Humbolt red in aniline oil and spirit, and decolourisation with spirit. The bacilli can also be demonstrated, but less satisfactorily, by Gram's method, and still less so by Weigert's solution of gentian violet. The inoculation of monkey3 with our cultivations has so far been unsuccessful in inducing syphilis, but so has also inoculation with portions of chancres and of phagedænic sores. Nevertheless, the cultivation of a morphologically peculiar bacillus in two instances from the blood of syphilitics and from some of the chief lesions, and its recognition in the diseased tissues, have from analogy a striking significance in regard to the etiology of syphilis, and lead us to hope that by the continuation of our investigation we may yet experimentally prove that the organism described is the cause or the carrier of the disease. Owing to the attention which this question is now exciting in Germany we considered it necessary to publish this preliminary notice of our results.

\section{A NOTE ON CYCLIC ALBUMINURIA.}

\section{BY WILLIAM COLLIER, M.A., M.D.,} PHYSICIAN TO THE RADCLIFFE INFIRMARY.

THE following case may prove of interest in connexion with Dr. Pavy's recent paper, reported in THE LANCET of March 6th, on Cyclic Albuminuria.

I was recently called to an undergraduate who was suffering from epistaxis. In answer to inquiries I learnt that for some years he had had at intervals similar attacks, which as a rule proved difficult to stop, though bleeding from other parts of the body, the result of cuts, or after the extraction of teeth, was easily controlled. The last attack of epistaxis occurred twelve monihs previously. He also 
mentioned that a brother and sister were at times troubled with epistaxis. The bleeding was on this occasion easily controlled by the use of alum. Two days later I was again sent for, and informed that the bleeding had returned early in the day and had been very troublesome. On asking for a little of his urine, the patient at once volunteered the following information. Three years ago, when at Rugby School, after getting a chill, albumen was found in his urine. Twelve months ago, when thinking of becoming a candidate for the Indian Civil Service, he underwent a medical examination, and was warned that he would probably be refused, as his urine contained albumen. In consequence of this advice he gave up the idea. Since the first appearance of albumen three years ago his general health had been good and he had led a very active life, having on two occasions run in the Rugby Crick (the annual longdistance cross-country steeplechase), and had gone in for long-distance running at Oxford. His condition was as follows:-Complexion pale; tongue clean; pulse slow and resisting. Heart: slight accentuation of second sound; no evidence of hypertrophy. Lungs healthy. Urine passed at the time of my visit (about 5 P.M.) was acid, sp. gr. 1030 , contained a large quantity of lithates, and with the nitric acid tests showed about one-eighth albumen. I requested him, as is my invariable custom, in accordance with Dr. George Johnson's teaching, to send me a specimen of urine passed the following morning immediately on rising. On testing this with the ordinary nitric acid tests I could find not the slightest trace of albumen, though the tests were carefully repeated several times. Another specimen, passed about I P.M. on the same day, contained about one-eighth albumen. The microscope showed the presence of amorphous urates, but no casts could be found. As the patient was feeling very ill, and had been for the past few days much troubled with headache, I advised him to return home. $\mathrm{He}$ left Oxford the same afternoon, and I have not as yet heard from him.

The case is very incomplete, and as I had only one opportunity of testing the morning urine, I should not have reported it had it not seemed to me to suggest that possibly some of the cases remarked on by Dr. Pavy may at the end of another year or two develop all the usual symptoms of chronic Bright's disease. I cannot doubt that, in spite of the patient's apparently sound health until within the last week or two, the headache, epistaxis, high-tensioned pulse, slightly accentuated second sound of heart, and albuminous urine from which he suffered, were all secondary to slow changes in the kidneys, the result of the chill taken when at Rugby three years previously. Nor is it improbable that had $I$ had frequent opportunities of examining his early morning urine, I should have found in such specimens, as in my former examination, an absence, or at the most a very faint trace, of albumen. On referring to my notes of the clinical lectures given by Dr. George Johnson while I was a student at King's College Hospital, I find the following remarks in a lecture delivered June, 1879, on Practical Matters connected with Bright's Disease:- "Remember, also, to test urine both after food and exercise, as it frequently happens that scarcely a trace appears in the urine voided before breakfast, while a large quantity may be present in that passed an hour or two after. Exercise may cause the presence of albumen when the urine is unaffected by food." In proof of this Dr. Johnson related the case of a medical man suffering from chronic Bright's disease who found albumen after exercise, but never after food if he rested. While house-physician to the Wolverhampton Hospital, in which district cases of acute and chronic Bright's disease are very common among the stokers and hammerers at the various ironworks, I had ample opportunity of testing the value of Dr. Johnson's teaching, and can recall more than one case in which the urine was most markedly influenced by rest, containing only the slightest traces in the early morning, but considerable quantities in the middle of the day. Such facts would suggest the danger of disregarding the continued presence of albumen in the urine at certain periods in the day, in spite of the absence of symptoms-unless, at any rate, it had existed a considerable number of years.

Swansea HospitaL. - The secretary of the hospital has received, from an anonymous donor, a cheque for $£ 600$ towards the funds of the charity. Upwards of $£ 3000$ have been received in a similar way during the past few years.

\section{EXCISION OF THE ENTIRE UTERUS FOR} CANCER.

WITH NOTES OF A SUCCESSFUL OPERATION,

BY CHAS. E. JENNINGS, F.R.C.S. ENG., M.S., ASSISTANT-SURGEON TO THE CANCER AND NORTH-WEST LONDON HOSPITALS

THE object of two lectures which I delivered at the Cancer Hospital this session was to advocate vaginal extirpation of the uterus, for early cancer of the cervix, as the operavion to be usually performed, to the exclusion of other methods of treatment, as soon as the diagnosis is established. History teaches us that in the past the mortality which attended excision of the entire uterus was so great that the operation could only be considered a desperate remedy to be adopted for desperate conditions; to-day it is struggling for a settled place in surgery, and for the near future why should not extirpation of the uterus be followed by a measure of success equal to that which now accompanies other severe but recognised surgical procedures on abdominal organs? From statistics contained in a valuable paper on extirparion of the uterus, presented to the Obstetrical Society by Dr. Wm. Duncan in January, 1885, it appears that of 137 cases of abdominal extirpation 38 recovered and 99 died, being a mortality of 72 per cent., but of 276 cases of vaginal extirpation 197 recovered and 79 died, being a deathrate of $28^{\circ} 6$ per cent. The former group of operations were divided among seventy-one and the latter among sixty-nine surgeons, and therefore it seems right to conclude that, except under some special circumstances, the vaginal should be preferred to the abdominal method of uterine excision. This conclusion is strengthened by the fact that many of the operations in the two categories were performed by the same. operators. For example: Martin performed 6 hysterectomies by the abdominal method, and all the patients died; he operated 60 times by the vaginal method, with 47 recoveries and 13 deaths. Of 8 abdominal extirpations of the uterus by Billroth, 3 recovered and 5 died; of 12 patients operated upon by the same surgeon by the vaginal method, 8 recovered and 4 died. Of 8 abdominal operations by Schroeder there were 3 recoveries and 5 deaths; whilst of 27 vaginal extirpations there were 19 recoveries and 8 deaths. Guided by these statistics, I elected to excise the uterus per vaginam in the following case, where the removal of the entire organ was the only means by which life could possibly be prolonged beyond a very limited period.

On Sept. 1st, 1885, Mrs. R - aged thirty-fire, who had been twice married, attended at the Cancer Hospital, complaining of an offensive, sanguineous vaginal discharge, associated with pelvic pain and other symptoms, of some month's' duration-viz., since the birth of her lastchild. One of her sisters was reputed to have died some years ago of "cancer of the bladder." Physical examination disclosed an infiltration of the cervix uteri with ulceration around the os externum. The disease being apparently well localised, it was decided to destroy the neoplasm and surrounding tissue with caustic potash. This was accordingly done on Sept. 5th ; several sticks of caustic potash were employed, the cervix being previously painted liberally with a solution of muriate of cocaine to mitigate the pain. After the separation of the sloughs bleeding returned, and the necessity for a greater destruction of the cervix being evident, the patient was anæsthetised on Sept. 15th. The chief portion of the diseased cervix was scraped away with a curette, posteriorly as far as the junction of the cervix with the vagina, and Pacquelin's thermo-cautery was applied freely to the raw surface. This operation appearing satisfactory, the woman was duly discharged and treated as an out-patient; but the pain and hæmorrhage having soon returned, she became confined to her bed at home, and at the request of Mr. Prowse, who had been summoned to attend her, I examined the patient in consultation with him on Oct. 28th. The general strength had then declined considerably, and there were marked signs of anæmia. The vagina was flaccid, the uterus enlarged and a little prolapsed. That portion of the anterior lip of the cervix which remained after the previous operations was infiltrated; the posterior lip was absent, so that the finger on being passed upwards along the posterior vaginal wall glided into a conical rugose cavity, whose apex seemed limited superiorly by the internal os. The speedy recurrence and the rapid growth of the neoplasm convinced 